\title{
Confirmation of translatability
}

\section{and functionality certifies the dual endothelin 1/VEGFsp receptor (DEspR) protein}

Victoria L. M. Herrera ${ }^{1,2}$, Martin Steffen ${ }^{3}$, Ann Marie Moran ${ }^{1,2}$, Glaiza A. Tan ${ }^{1,2}$, Khristine A. Pasion ${ }^{1,2}$, Keith Rivera ${ }^{4}$, Darryl J. Pappin ${ }^{4}$ and Nelson Ruiz-Opazo ${ }^{1,2^{*}}$

\begin{abstract}
Background: In contrast to rat and mouse databases, the NCBI gene database lists the human dual-endothelin1/ VEGFsp receptor (DEspR, formerly Dear) as a unitary transcribed pseudogene due to a stop [TGA]-codon at codon\#14 in automated DNA and RNA sequences. However, re-analysis is needed given prior single gene studies detected a tryptophan [TGG]-codon\#14 by manual Sanger sequencing, demonstrated DEspR translatability and functionality, and since the demonstration of actual non-translatability through expression studies, the standard-of-excellence for pseudogene designation, has not been performed. Re-analysis must meet UNIPROT criteria for demonstration of a protein's existence at the highest (protein) level, which a priori, would override DNA- or RNA-based deductions.
\end{abstract}

Methods: To dissect the nucleotide sequence discrepancy, we performed Maxam-Gilbert sequencing and reviewed 727 RNA-seq entries. To comply with the highest level multiple UNIPROT criteria for determining DEspR's existence, we performed various experiments using multiple anti-DEspR monoclonal antibodies (mAbs) targeting distinct DEspR epitopes with one spanning the contested tryptophan [TGG]-codon\#14, assessing: (a) DEspR protein expression, (b) predicted full-length protein size, (c) sequence-predicted protein-specific properties beyond codon\#14: receptor glycosylation and internalization, (d) protein-partner interactions, and (e) DEspR functionality via DEspR-inhibition effects.

Results: Maxam-Gilbert sequencing and some RNA-seq entries demonstrate two guanines, hence a tryptophan [TGG]-codon\#14 within a compression site spanning an error-prone compression sequence motif. Western blot analysis using anti-DEspR mAbs targeting distinct DEspR epitopes detect the identical glycosylated $17.5 \mathrm{kDa}$ pulldown protein. Decrease in DEspR-protein size after PNGase-F digest demonstrates post-translational glycosylation, concordant with the consensus-glycosylation site beyond codon\#14. Like other small single-transmembrane proteins, mass spectrometry analysis of anti-DEspR mAb pull-down proteins do not detect DEspR, but detect DEspR-protein interactions with proteins implicated in intracellular trafficking and cancer. FACS analyses also detect DEspR-protein in different human cancer stem-like cells (CSCs). DEspR-inhibition studies identify DEspR-roles in CSC survival and growth. Live cell imaging detects fluorescently-labeled anti-DEspR mAb targeted-receptor internalization, concordant with the single internalization-recognition sequence also located beyond codon\#14.

Conclusions: Data confirm translatability of DEspR, the full-length DEspR protein beyond codon\#14, and elucidate DEspR-specific functionality. Along with detection of the tryptophan [TGG]-codon\#14 within an error-prone compression site, cumulative data demonstrating DEspR protein existence fulfill multiple UNIPROT criteria, thus refuting its pseudogene designation.

Keywords: DEspR, Pseudogene, DEspR protein-protein interactions

\footnotetext{
*Correspondence: nruizo@bu.edu

${ }^{1}$ Whitaker Cardiovascular Institute, Boston University School of Medicine,

700 Albany Street, Boston, MA 02118, USA

Full list of author information is available at the end of the article
} 


\section{Background}

In contrast to rat and mouse databases listing Dear as a gene, DNA and RNA sequence databases list the human Dear gene or the dual-endothelin1/VEGFsp receptor (DEspR) as a pseudogene [1] (Additional file 1: Figure S1). Automated DNA sequence databases report a stop codon [TGA] instead of tryptophan [TGG] at codon\#14 reported in single gene study [2]. The current NCBI pseudogene annotation updated in May 2016 and referenced in other sites is discrepant with the single research group single-gene studies of human DEspR showing expression in human kidney via immunohistochemistry using a polyclonal anti-DEspR antibody, and functional studies of human DEspR expressed in permanent Cos1 cell transfectants detecting the predicted protein size by Western blot analysis as well as binding to DEspR-ligands (endothelin-1 and VEGFsp) [2]. The NCBI pseudogene annotation is also discrepant with the single gene study demonstrating DEspR-specific functional roles in cancer and putative regulation at the splicing level with detection of both unspliced and spliced DEspR RNA in human tumor cells by allele-specific amplification-refractory mutation system (ARMS) methodology [3].

Experimental clarification is warranted since the basis for the NCBI pseudogene annotation, automated DNA/ RNA-sequencing, is known to have reproducible systematic sequencing errors, regardless of technology [4]. Occurrences of, hence risks for, systematic errors eliminate the a priori assumption that multiple occurrences negate errors. More specifically, systematic errors in high throughput DNA sequencing has been observed to occur "even in overlapping paired reads from high-coverage data, approximately one in $1000 \mathrm{bp}$, and are highly replicable across experiments" [4]. Moreover, given discrepancies among methodologies, the Sanger sequencing is the final determinant of sequence discrepancy since "any difference from the Sanger sequence is defined as a sequencing error" [5].

Further support for the need for scientific clarification is found in the GENCODE Pseudogene Resource which states that "the definition of a pseudogene is based on the presence of specific characteristics such as premature stop codon, coding sequence frame shift, truncation, or disabling insertion/deletion-unless evidence (transcriptional, functional, publication) shows that the locus represents a protein-coding gene" [6]. Concordant with Pei et al. [6], Kageyama et al. [7] explicitly states that "before a particular transcript can be determined to be a long noncoding RNA (or 'transcribed pseudogene'), there must be somewhat convincing evidence for its lack of translatability." Hence, deductions from automated sequence databases need to be evaluated in the context of experimental evidence for translatability and functionality.
To address the need for scientific clarification of DEspR (Dear) as a gene or pseudogene, we therefore upheld established standards that (1) "the translatability of the candidate can be validated with specific antibodies against amino acid sequences predicted from the ORF," [7] and that (2) "an assessment of ... a protein's molecular activity by biochemical methods should be the final certification of an active gene product" [7]. These perspectives are codified in the Central Protein Resource UNIPROT criteria for translatability: "evidence for existence of a protein at the protein level, such as via antibody detection, is the highest level of evidence" [8].

Here we confirm the existence of the DEspR gene product at the nucleotide and protein level. We show that both Sanger and Maxam-Gilbert sequencing detect two G's for a tryptophan [TGG]-codon\#14. We also determine that some RNA-seq entries also contain two G's but with an extra A or TA, as the region in question spans a canonical Yamakawa compression motif [9]. Monoclonal anti-DEspR antibodies to different DEspR epitopes detect the identical glycosylated $17.5 \mathrm{kDa}$ pull-down protein from membrane-bound proteins from human tumor cells and the Cos1-DEspR + permanent transfectants cells. More importantly, DEspR molecular activity, protein-protein interactions, protein-specific properties and functionality are shown, and found to play key roles in cancer stem cell anoikis resistance and growth.

\section{Results}

\section{Confirmation of two G's and compression site}

To determine whether the two G's are detected forming tryptophan [TGG]-codon\#14, as observed in Sanger sequencing (Additional file 2: Figure S2), we performed manual Maxam-Gilbert sequencing using $8 \%$ denaturing polyacrylamide sequencing gels on ${ }^{32} \mathrm{P}$ end-labeled PCR-amplified cDNA electrophoresed at three different fixed wattages (Fig. 1a). Clearly, the two G's are noted with the corresponding $\mathrm{C}$ bands that accompany most $\mathrm{G}$ nucleotides. Interestingly, the compression in the gel run is consistently observed in Sanger sequencing and in Maxam-Gilbert sequencing, and contains the compression motif found in $68 \%$ of sequencing errors with compression [9] (Fig. 1a; Additional file 2: Figure S2). This compression results in the "slippage' of the two G's with a $\mathrm{T}$ nucleotide, thus leaving a space in the gel read. Additionally, the compression region is within a 3-nt stem-loop structure that could render this region prone to sequencing errors which are not present in rat and mouse DEspR gene sequences, both of which also contain the identical tryptophan [TGG]-codon\#14 (Additional file 2: Figure S2).

Importantly, queries against subsets of the NCBI sequence read archive (SRA) database reveals DEspR-specific exon sequences which would not be expected for the 

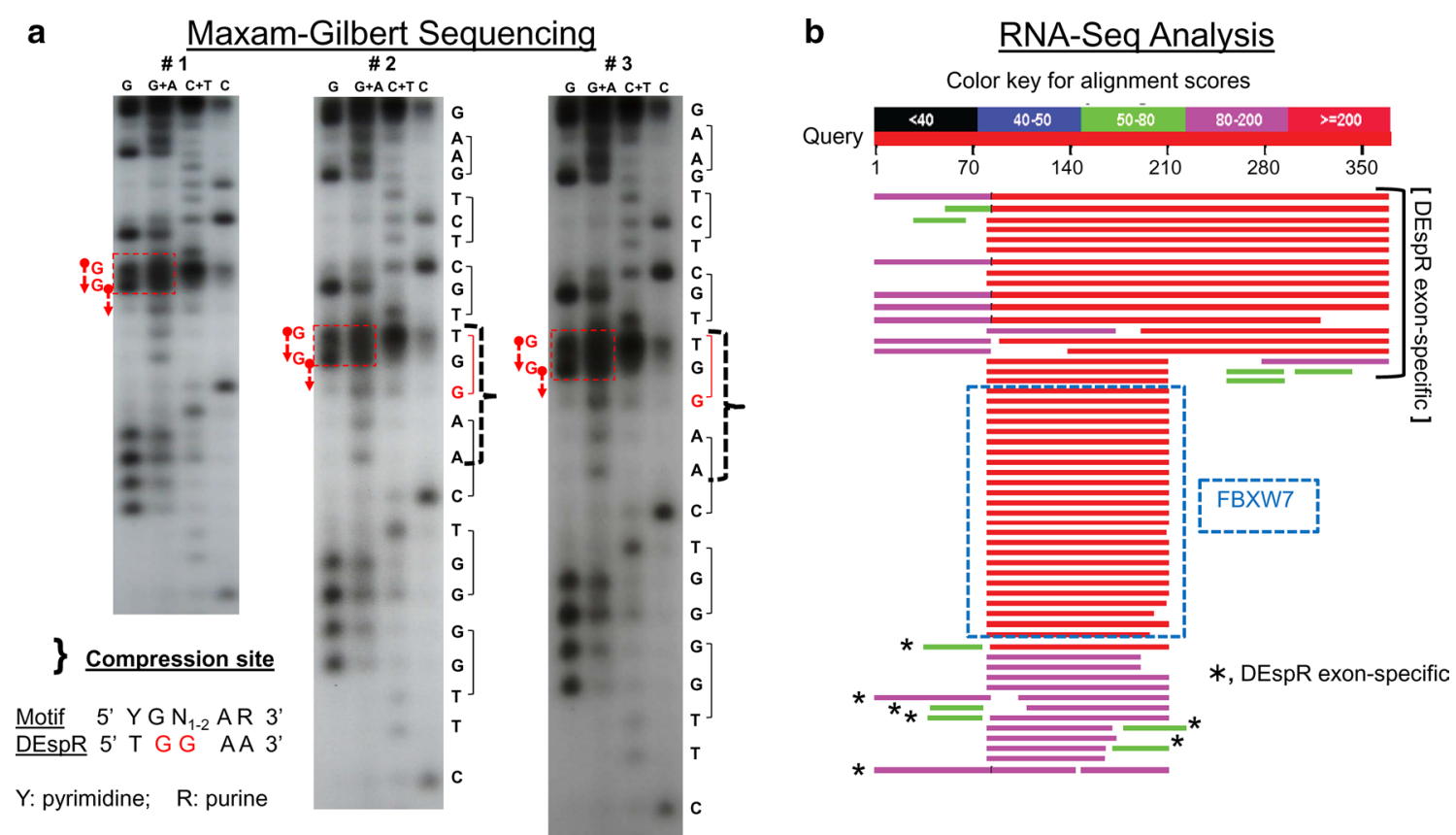

Fig. 1 DEspR DNA and RNA sequence analysis. a Maxam-Gilbert DNA nucleotide sequence analysis in three different gel runs of increasing wattage ( $\# 1=25$ watts; $\# 2=35$ watts; \#3 = 50 watts) spanning controversial region shows: consistent area of compression ( 3 ) which contains the Yamakawa compression-motif. The compressed two G's and single T are depicted in codon\#14:TGG.], codons; the two G's (in red). b Representative RNA-Seq analysis of 727 unedited RNA-seq entries show DEspR exon-specific RNAs distinguished from the anti-sense strand transcript, FBXW7, exon-specific sequence. Query DEspR sequence spans 1-372 nt of DEspR transcript

FBXW7 transcript in the opposite strand (Fig. 1b). Notably, five entries show the two G's out of 124 data entries in said file (Additional file 3: Figure S3), and that these two G's are associated with an insertion of a "T-A' or an "A" (Additional file 3: Figure S3). These observations show that the two G's are indeed detected and are located within a problematic sequencing region, concordant with observations in manual sequencing gels of nucleotide compression spanning a known sequence motif for compression [9]. These data show the questioned two G's in support of tryptophan [TGG] codon in 5/124 sequences similar to manual sequencing runs. Given that Sanger-sequencing is the accepted standard final determinant of nucleotide discrepancies [5], these experimental data support the need for clarification and demonstration of DEspR protein expression.

\section{Analysis of translatability and protein-specific properties} In order to clarify the existence of DEspR protein complying with established UNIPROT criteria for detection of protein by antibody made from deduced amino acid sequences, we performed anti-DEspR mAb pull-down and subsequent Western blot and mass spectrometry analyses of pull-down products from cell membranes. Membranes were isolated from human glioblastoma tumor cells and U87 cancer stem-like cells (CSC) which we isolated and characterized for CSC tumor initiating properties [3]. We performed pull-down experiments using an anti-human DEspR specific antibody, 5g12e8 mAb (Fig. 2a), which detected DEspR on prior Western blot analysis of CSC membrane proteins [3].

Analysis of pull-down products by mass spectrometry (MS) (Table 1; Additional file 4: Table S1) revealed that the DEspR-protein interacts with several proteins involved in intracellular trafficking, angiogenesis, and/or cancer: vimentin, Gal-3, Gal-1 and TMED10. Although MS analyses did not detect DEspR (Table 1), Western blot analyses of pull-down products detected DEspR protein bands at $\sim 17.5$ and $\sim 12.5 \mathrm{kDa}$, larger than the deduced amino acid sequence predicting $\sim 10 \mathrm{kDa}$, and distinct from the other proteins in the pull-down-complex: Rab1b (22 kDa), Galectin-1 (14 kDa) and TMED10 (25 kDa) (Fig. 2b). Data indicate that DEspR is likely glycosylated given its consensus glycosylation site [N-F-S-G] (Fig. 2a), and clearly distinct from the other proteins in the pulldown complex as evidenced by size, relative abundance, and the lack of antibody cross-reactivity (Fig. 2b). Glycosylation of DEspR is confirmed after peptide- N-glycosidase F (PNGase F) treatment of pull-down proteins showing decrease in size of DEspR upon Western blot 

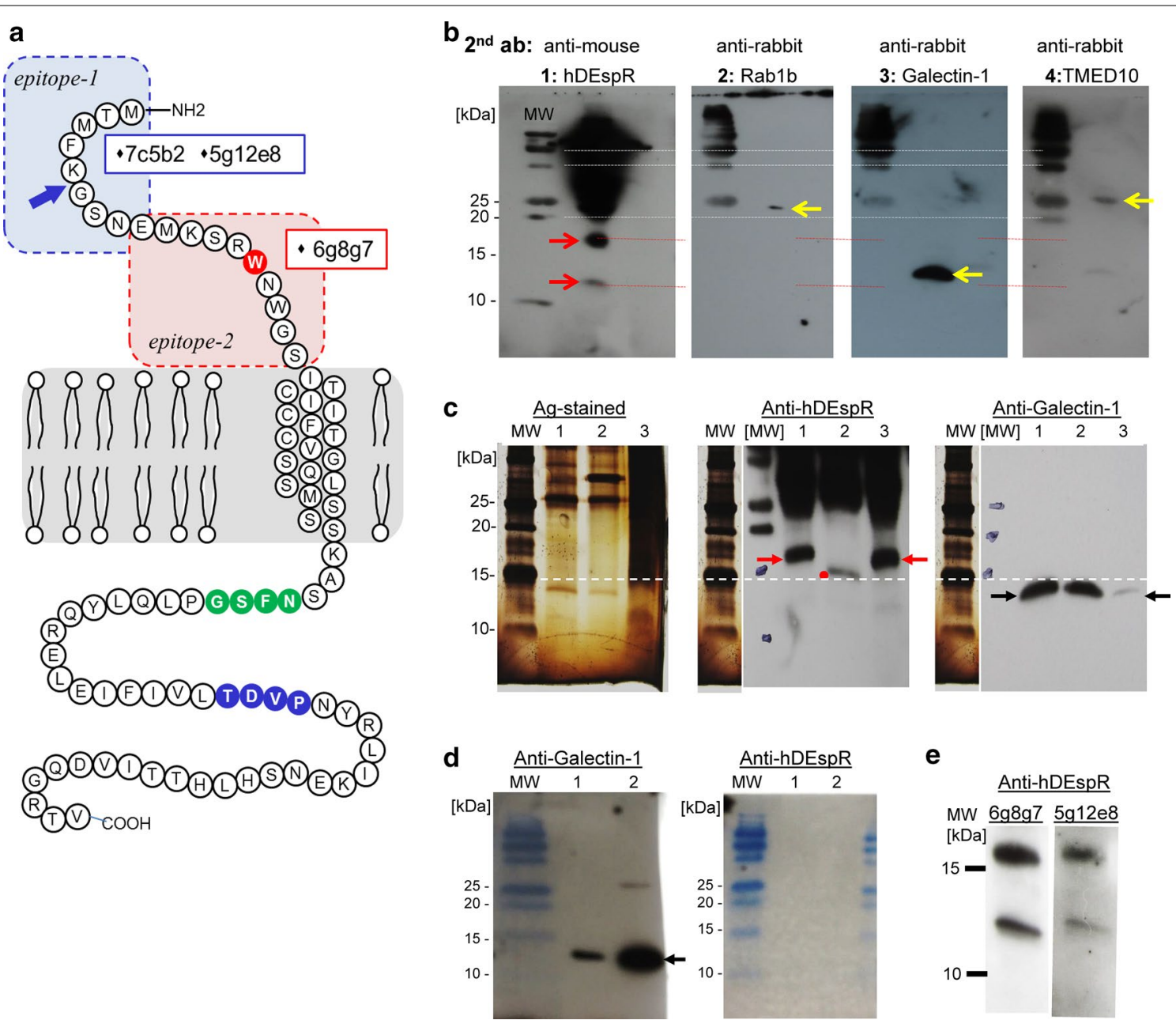

Fig. 2 Analysis of DEspR translatability. a Schematic diagram of DEspR protein and mAb-epitopes. Two distinct peptides (epitope-1, epitope-2) in the extracellular domain were used to develop murine monoclonal antibodies (mAbs). Two high-affinity mAbs target human-specific epitope-1: 7c5b2, $5 \mathrm{~g} 12 \mathrm{e} 8$; and one high-affinity mAb targets the pan-species reactive epitope-2, identical in human, monkey, and rat. Epitope-2 spans the putative ligand binding domain [24]. The $5 \mathrm{~g} 12 \mathrm{e} 8 \mathrm{mAb}$ was used in pull-down experiments; $5 \mathrm{~g} 12 \mathrm{e} 8$ and $6 \mathrm{~g} 8 \mathrm{~g} 7$ were used in Western blot analyses, 7c5b2 $\mathrm{mAb}$ was used in FACs analysis, immunostaining, and internalization assays, and all three were used in functional inhibition assays. The contested tryptophan (W)-aa\#14 (red); consensus glycosylation site sequence: (green, N-F-S-G), known internalization recognition sequence: (blue: T-D-V-P). A blue arrow marks the splice junction between exon1 and exon2, i.e. between amino-acids G and K (aa\#5-\#6). b Sequential Western blot analyses of pull-down proteins from glioblastoma U87 membrane proteins using different antibodies specific for proteins identified by mass spectrometry analysis of pull-down protein-products. The identical blot was sequentially probed, stripped of antibody, confirmed as stripped, then re-probed in the following order: \#1: anti-hDEspR-5g12e8 mouse mAb, \#2: anti-Rab1b rabbit polyclonal Ab (pAb), \#3: anti-Galectin-1 rabbit pAb; \#4: anti-TMED10 rabbit pAb. Molecular weight markers are noted. DEspR bands are 17.5 and 12.5 kDa. Expected sizes are detected for Rab1 b: 22 kDa, Galectin-1: $14 \mathrm{kDa}$, and TMED10: $25 \mathrm{kDa}$. c Panel-1 shows silver-stained gel of pull-down protein products using $5 \mathrm{~g} 12 \mathrm{e} 8 \mathrm{mAb}$ from membrane proteins isolated from: (1) glioblastoma U87 CSCs, (2) PNGase-digested sample of pull-down proteins from U87 CSCs, (3) permanent transfectants DEspR-positive Cos1-cells. Panel-2 shows Western blot analysis using anti-DEspR 5g12e8 mAb showing DEspR band (lane 1), smaller DEspR + band after PNGase digest-samples (lane 2), and identically-sized DEspR band in DEspR-positive Cos1-cell permanent transfectants showing appropriate splicing and translatability of DEspR-minigene transfected into Cos-1 cells (lane 3). Panel-3 Western blot analysis of different wells in the same gel run probed with anti-Galectin-1 pAb showing distinct sized protein bands, thus confirming DEspR-specific bands are not Galectin-1 protein bands, and that Galectin-1 is not glycosylated as reported. d Western blot analysis of Galectin-1 recombinant protein. Panel 1 overlay of gel-image and western blot image showing detection of Galectin-1 recombinant protein at expected size $14 \mathrm{kDa}$. Panel 2 overlay of gel-image and western blot image probed with anti-DEspR 5g12e8 mAb showing non-cross reactivity of anti-hDEspR mAb with Galectin-1. e Sequential western blot analysis of 5g12e8pull-down proteins from U87 CSCs probed first with $6 \mathrm{~g} 8 \mathrm{~g} 7$ (left panel), and subsequently with $5 \mathrm{~g} 12 \mathrm{e} 8$ after 'stripping' (right panel), detects identical protein bands. This confirms that $6 \mathrm{~g} 8 \mathrm{~g} 7$ epitope is on the same protein as $5 \mathrm{~g} 12 \mathrm{e} 8 \mathrm{epitope}$, thus corroborating DEspR protein existence 
Table 1 Proteins pulled-down with anti-DEspR mAb 5g12e8 from U87 CSC membranes

\begin{tabular}{|c|c|c|c|c|c|c|c|}
\hline Accession & $\%$ & \#SM & \#P & \#AAs & Mass & Score & Description \\
\hline P08670 & 55.8 & 36 & 31 & 466 & 53,619 & 177.59 & Vimentin (VIM) \\
\hline P60709 & 34.4 & 11 & 9 & 375 & 41,709 & 66.79 & Actin, cytoplasmic 1 (ACTB) \\
\hline P68371 & 41.6 & 13 & 12 & 445 & 49,799 & 56.53 & Tubulin beta-4B chain (TUBB4B) \\
\hline P07437 & 35.1 & 11 & 10 & 444 & 49,638 & 47.84 & Tubulin beta chain (TUBB) \\
\hline Q13885 & 29.0 & 10 & 9 & 445 & 49,874 & 42.11 & Tubulin beta-2A chain (TUBB2A) \\
\hline P17931 & 25.6 & 7 & 5 & 250 & 26,136 & 39.26 & Galectin-3 (LGALS3) \\
\hline P09382 & 49.6 & 7 & 6 & 135 & 14,706 & 38.90 & Galectin-1 (LGALS1) \\
\hline Q13509 & 25.6 & 8 & 8 & 450 & 50,400 & 32.86 & Tubulin beta-3 chain (TUBB3) \\
\hline Q9BUF5 & 15.7 & 6 & 5 & 446 & 49,825 & 21.46 & Tubulin beta- 6 chain (TUBB6) \\
\hline P61006 & 13.0 & 2 & 2 & 207 & 23,653 & 12.01 & Ras-related protein Rab-8A (RAB8A) \\
\hline C9J8S3 & 15.0 & 2 & 2 & 160 & 18,015 & 11.01 & Ras-related protein Rab-7a (RAB7A) \\
\hline P61026 & 6.0 & 1 & 1 & 200 & 22,526 & 9.59 & Ras-related protein Rab-10 (RAB10) \\
\hline P51153 & 5.9 & 1 & 1 & 203 & 22,759 & 7.60 & Ras-related protein Rab-13 (RAB13) \\
\hline HOYNE9 & 6.4 & 1 & 1 & 188 & 21,854 & 7.51 & Ras-related protein Rab-8B (Fragment) (RAB8B) \\
\hline G3V2K7 & 7.2 & 1 & 1 & 153 & 16,893 & 5.96 & Transmembrane emp24 domain-containing protein $10(T M E D 10)^{2}$ \\
\hline Q9Y3B3-2 & 5.3 & 1 & 1 & 188 & 21,219 & 4.85 & Transmembrane emp24 domain-containing protein 7 (TMED7) \\
\hline Q9BVK6 & 3.8 & 1 & 1 & 235 & 27,260 & 4.36 & Transmembrane emp24 domain-containing protein 9 (TMED9) \\
\hline F8WBC0 & 34.4 & 1 & 1 & 32 & 3501 & 2.90 & Ras-related protein Rap-1b (Fragment) (RAP1B) \\
\hline
\end{tabular}

Proteins in italic face letters are proteins detected in three independent pull-down experiments

$\%$ the percentage of the protein sequence covered by identified peptides; \#SM the number of peptide spectrum matches; \#P the total number of distinct peptide sequences identified in the protein group; \#AAs number of amino acids; Mass, mass in Daltons; Score, the sum of the ion scores of all peptides that were identified

a Proteins verified by Western blot analysis of pull-down proteins; Rab-1b was detected in two independent experiments and verified by Western blot analysis (Fig. 2b)

analysis (Fig. 2c, middle panel, lane 2). These data validate the canonical glycosylation site beyond the purported pseudogene stop codon\#14, and provides a mechanism for DEspR-Galectin-1 and DEspR-Galectin-3 binding as observed in the pull-down products.

However, because of the abundance of Galectin-1 in the pull-down products, we performed more Western blot analyses to eliminate the possibility that anti-DEspR antibody cross reacts with Galectin-1 and vice versa. As shown in Fig. 2c, the anti-human DEspR 5g12e8 mAb detects the $17.5 \mathrm{kDa}$ glycosylated DEspR in the pulldown products from U87-CSC cell membrane proteins and from the membrane proteins isolated from DEspRpositive Cos1-permanent transfectant cells. These DEspR + Cos1-permanent transfectants cells were previously shown to express human DEspR by immunostaining, and to competitively bind ligands and anti-DEspR (7c5b2) mAb [2]. After PNGase F digestion, 5g12e8 also detected the smaller, hence deglycosylated DEspR (Fig. 2c, middle panel). In contrast, anti-Galectin-1 mAb detected a $14 \mathrm{kDa}$ protein band with markedly different size and expression levels to DEspR in all three lanes (Fig. 2c, right panel).

To further confirm this distinction, we performed double Western blot analyses of Galectin-1 recombinant protein. As shown in Fig. 2d, anti-Galectin-1 mAb detected human Galectin-1, in contrast to the anti-human DEspR $5 \mathrm{~g} 12 \mathrm{e} 8 \mathrm{mAb}$ which did not cross react to both 100 and $500 \mathrm{ng}$ of purified recombinant human Galectin-1 on Western blot analysis, thus confirming that DEspR in the pull-down product is distinct from other pull-down products (Fig. 2b) especially Galectin-1 (Fig. 2c). Additionally, since Galectin-1 is known not to be glycosylated [10] as confirmed in Fig. 2c, the PNGase F-treated sample showing a decrease in size cannot, therefore, be Galectin-1.

To further corroborate the detection of human DEspR protein in human tumor cells, we performed sequential probing of the identical Western blot with two different anti-human DEspR mAbs targeting different epitopes. As shown in Fig. 2e, the 6g8g7 mAb targeting the epitope spanning the disputed tryptophan [TGG]-codon\#14 (Fig. 2a) detects both the glycosylated $17.5 \mathrm{kDa}$ and the less glycosylated $12.5 \mathrm{kDa}$ DEspR bands. After removal of the $6 \mathrm{~g} 8 \mathrm{~g} 7$ antibody and demonstration of no residual signals, the Western blot was re-probed with the $5 \mathrm{~g} 12 \mathrm{e} 8$ $\mathrm{mAb}$ used in the pull-down experiment and which targets a human DEspR-specific epitope spanning a peptide present only if correctly spliced (Fig. 2a). This 5g12e8 probed western blot detected the identical protein bands (Fig. 2e), thus showing that two different anti-hDEspR mAbs 6g8g7 and 5g12e8, which target different epitopes, 
detect the same protein bands representing spliced and glycosylated DEspR.

\section{Protein-protein interactions: analysis of co-localization of DEspR and Galectin-1}

Since both DEspR and Galectin-1 are "pulled-down" consistently $(n=6)$, we next determined whether they are co-localized in tumors. We performed double immunostaining of xenograft tumors derived from U87-CSCs [3] using human specific anti-human DEspR mAb 7c5b2 and human-specific anti-Galectin-1 antibody. As shown in Fig. 3a, DEspR and Galectin-1 are indeed co-localized in tumors, and located more in the expanding tumor zone. Additionally, as shown in Fig. 3b, DEspR and Galectin-1 are co-expressed in tumor cells that have invaded through the xenograft tumor cap and adhered onto a microvessel in the surrounding subcutaneous tissue of the host nude rat. The detection of co-localization is concordant with the pull-down of both DEspR and Galectin-1 in multiple independent experiments (Table 1).

\section{Analysis of functionality at multiple levels certifies DEspR protein}

While we showed DEspR functionality in human tumor cells by showing that DEspR inhibition via the $7 \mathrm{c} 5 \mathrm{~b} 2 \mathrm{mAb}$ inhibited angiogenesis, tumor growth and invasiveness [3], the NCBI pseudogene designation persists (updated in May 2016). In addition to manual nucleotide DNA sequencing (Fig. 1a; Additional file 2: Figure S2), review of RNA-Seq sequence entries (Fig. 1b; Additional file 3: Figure S3), and detection of DEspR translatability or expression (Figs. 2, 3 ), to provide further evidence against the pseudogene designation, we further studied DEspR functionality since demonstration of functionality of a protein certifies that protein's existence [7]. To ascertain functionality, we used different anti-human DEspR mAbs whose epitopes are depicted in Fig. 2a: 7c5b2, 5g12e8 and 6g8g7 (Fig. 4), and tested for DEspR in different cancer tissue types.

As shown in Fig. 4, we studied DEspR-inhibition effects on anoikis resistant cancer stem-like cells (CSCs) isolated from human U87 glioblastoma tumor cell line [3], human MDA-MB-231 triple negative breast cancer cell line, human H460 non-small cell lung cancer cell line and human Panc1 pancreatic ductal adenocarcinoma cell line [3]. To first demonstrate that DEspR protein is expressed on the different CSCs, we performed FACS analysis using AF568-fluorescently labeled 7c5b2 antiDEspR mAb (Fig. 2a). Figure 4a shows that, compared to isotype control, fluorescently labeled $7 \mathrm{c} 5 \mathrm{~b} 2-\mathrm{mAb}$ specifically detected DEspR on the cell membrane in $\sim 50 \%$ of U87-CSCs, $\sim 60 \%$ of MB-231-CSCs, $71 \%$ of H460CSCs and $\geq 55 \%$ of Panc1-CSCs in the suspension culture conditions used.
To demonstrate the functionality of DEspR proteins present on the cell membrane, in vitro inhibition studies were performed using the $7 \mathrm{c} 5 \mathrm{~b} 2,5 \mathrm{~g} 12 \mathrm{e} 8$ and $6 \mathrm{~g} 8 \mathrm{~g} 7$ anti-human DEspR mAbs which target different DEspR epitopes (Fig. 2a). As shown in Fig. 4b, all three mAbs significantly inhibited CSC growth in the four cancer tissue type-CSCs tested $(\mathrm{P}<0.0001$, One Way ANOVA with Tukey's multiple comparisons test), in contrast to non-treated contemporaneous controls respectively. We note that CSC growth is representative of survival in suspension culture, proliferation and ability to form stemcell like spheroids in suspension culture conditions. We also note some variations in the inhibition of CSC growth in vitro by the different $\mathrm{mAbs}$, with $7 \mathrm{c} 5 \mathrm{~b} 2$ an $6 \mathrm{~g} 8 \mathrm{~g} 7$ performing better than $5 \mathrm{~g} 12 \mathrm{e} 8$ (Fig. 4), but that all mAbs significantly inhibit survival, proliferation, and spheroid formation (CSC-growth) of U87-CSCs, MDA-MB-231CSCs, H460-CSCs and Panc1-CSCs.

To further demonstrate DEspR functionality, we performed live-cell imaging of Panc1 cells using AF568fluorescently labeled 7c5b2 mAb (Fig. 5) given the single consensus internalization-recognition sequence [T-D$\mathrm{V}-\mathrm{P}]$ in the deduced DEspR amino acid protein located beyond the contested stop codon $v s$ amino acid \#14-W (Fig. 2a). Demonstration of internalization would indicate translation and expression of the DEspR protein beyond the purported [TGA] stop codon\#14, thus refuting the presence of the stop codon. As shown in Fig. 5a, time series of live-cell imaging shows increasing amounts of intracellular internalization beginning around $15-30 \mathrm{~min}$ and incrementally increasing up to $75 \mathrm{~min}$ after addition of fluorescent 7c5b2 anti-DEspR mAb. Higher magnification confirms intracellular fluorescence accumulation with fluorescently labeled 7c5b2 but not with isotype control IgG2b-AF568 (Fig. 5b), thus indicating AF568labeled 7c5b2-DEspR-mediated internalization rather than non-specific endocytosis. Demonstration of internalization affirms DEspR protein functionality, and further refutes the presence of the stop codon.

\section{Discussion \\ Stop [TGA] codon vs tryptophan [TGG] codon within a sequence-compression region}

The detection of the two G's to make tryptophan [TGG]codon\#14 in manual sequencing Sanger-dideoxy sequencing and Maxam and Gilbert sequencing, along with confirmation of sequence compression at the site within a Yamakawa compression-motif whether sequencing the sense strand (Maxam-Gilbert) or the antisense strand (Sanger dideoxy sequence), strongly suggests that the NCBI designation of human Dear/DEspR as a pseudogene based on stop codon\#14 is erroneous. Following the accepted standard that manual Sanger sequencing defines 
a
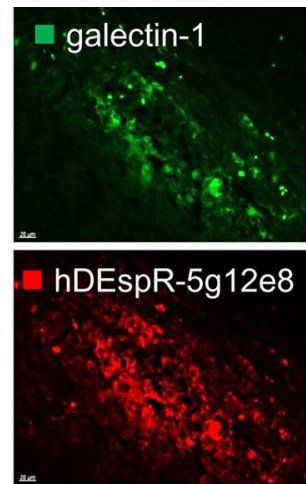

DAPI

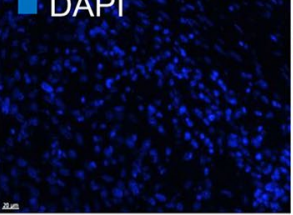

b $\mathrm{hDEspR-5g12e8}$

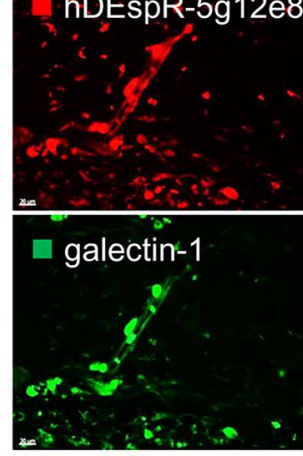

DAPI

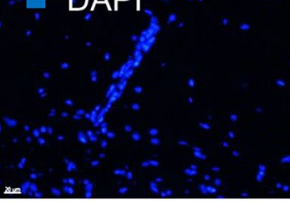

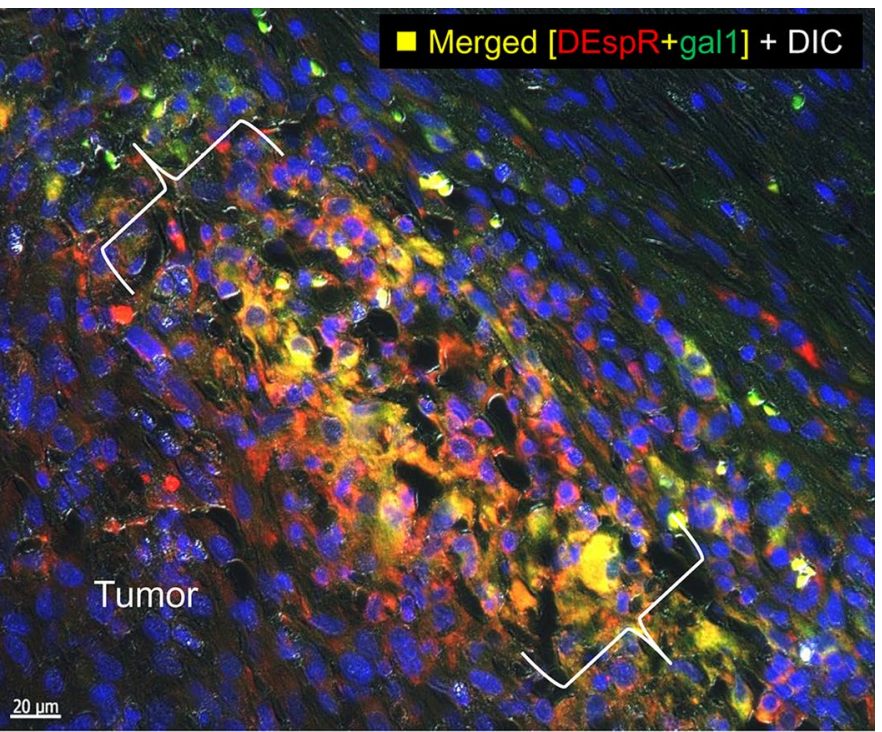

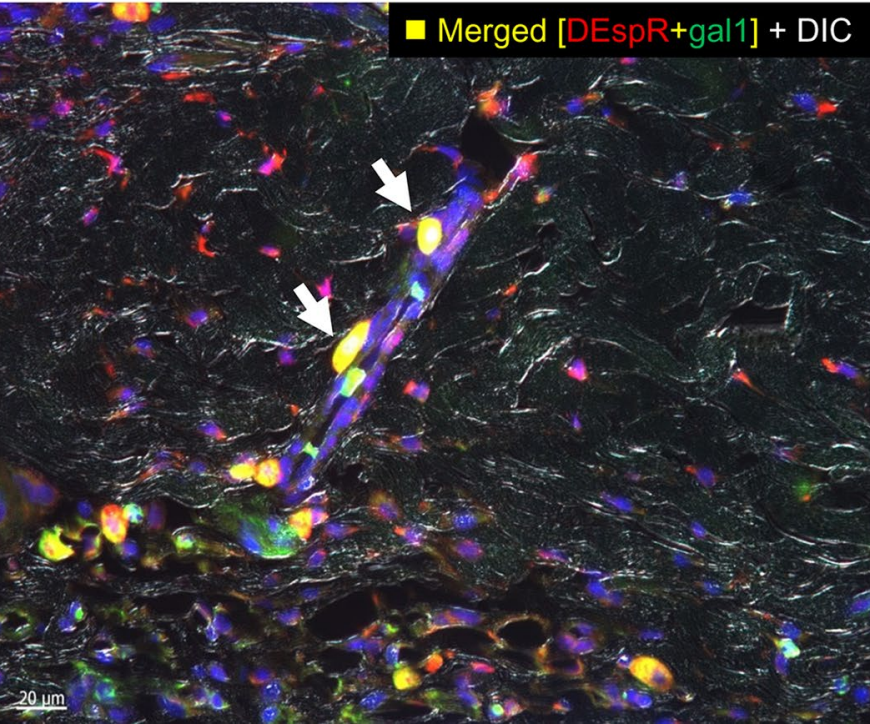

Fig. 3 Dual-fluorescence co-immunostaining analysis of DEspR and Galectin-1 expression. a Analysis of the expanding tumor zone of a U87-CSC xenograft tumor invading through the tumor fibrous cap. Co-localization (yellow, yellow dotted circle) of increased human-DEspR expression (red dotted circle) in invasive U87 tumor cells and Galectin-1 (green dotted circle) is observed in the invasive front. \{\}, invasive tumor front. Host subcutaneous tissue is to the upper right corner. b Co-localization of hDEspR and Galectin-1 is detected in tumor cells adhering to the outer wall of a microvessel in the subcutaneous tissue demonstrating invasive nature of $U 87$ in xenograft tumors and homing to microvessels

the correct sequence rather than the automated sequence, the single gene analysis data indicates that the TGG codon is present rather than the TGA stop codon reported in automated DNA sequencing databases. This interpretation is supported by the fact that systematic sequencing errors are documented to occur with both position- and sequence-specificity even in "next generation sequencing technologies at rates greater than prior technologies" [4]. Existence of the tryptophan[TGG]-codon\#14 is independently supported by detection of the DEspR protein by
mAbs 5g12e8 and 7c5b2 whose peptide epitopes exist only if the DEspR RNA is spliced, and in the case of $6 \mathrm{~g} 8 \mathrm{~g} 7$, only if the contested tryptophan[TGG]-codon\#14 is present (Fig. 2a). Notably, 5g12e8 and 6g8g7 detect DEspR-protein as a glycosylated $12.5-17.5 \mathrm{kDa}$ protein band, much greater than the $1.5 \mathrm{kDa}$ predicted from a 13 amino acid peptide should there be a stop codon at codon\#14. Furthermore, functional validation of the glycosylation and internalization recognition sequences beyond codon\#14 (Figs. 2, 5) experimentally refute a stop codon at codon\#14. 


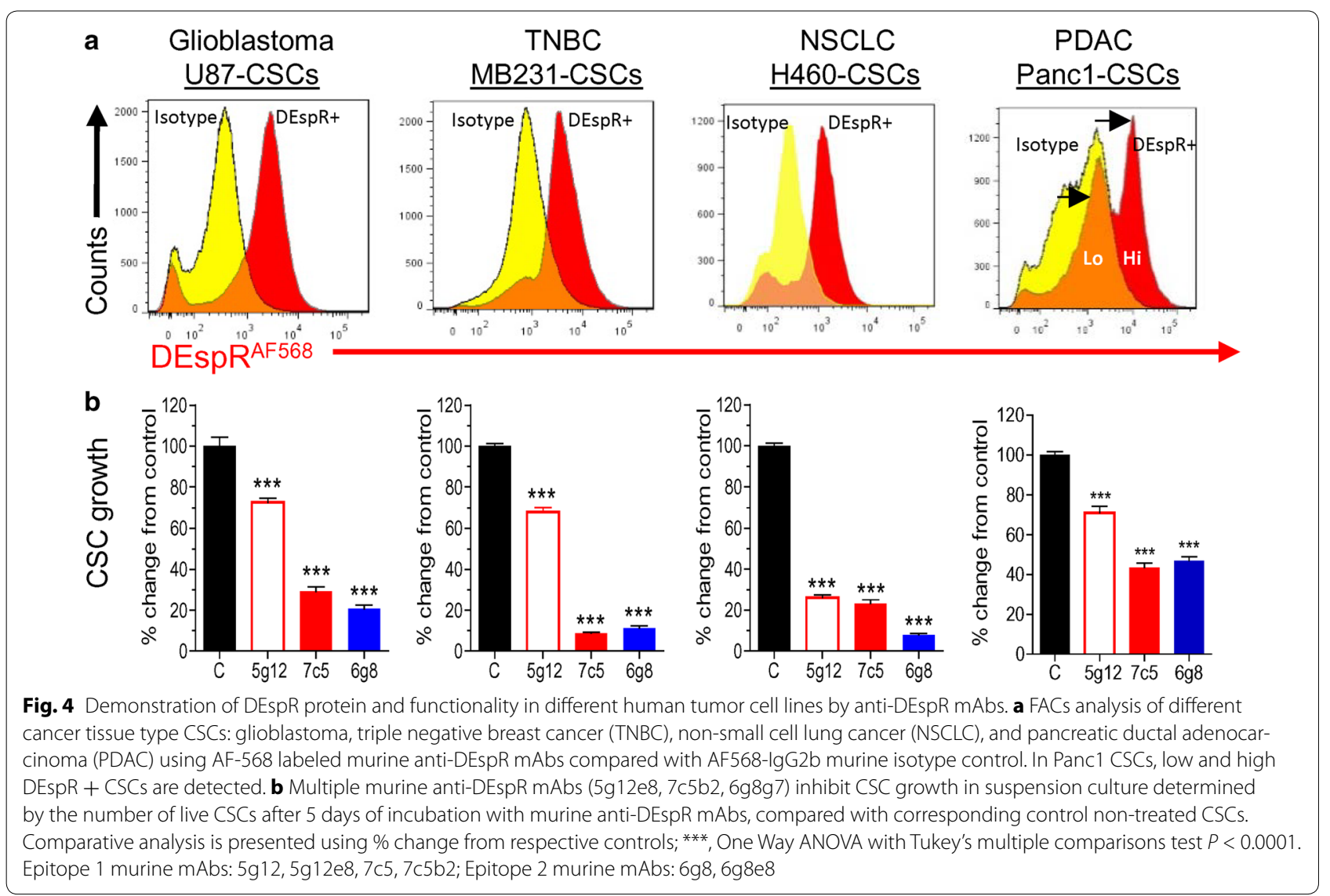

Insights regarding other high throughput database reports The non-detection of DEspR sequences in normal human tissue RNA-Seq databases is unsurprising since DEspR is not expressed or minimally expressed in normal tissues. Moreover, detection of unspliced DEspR-specific RNA as the dominant species [3] does not indicate non-translation, since regulation at the splicing level has recently been described for granulocyte differentiation whereby the dominant RNA species in the cell is the unspliced form $[11,12]$, similar to observations for detection of both spliced and unspliced DEspR-transcripts by ARMSPCR [3].

Moreover, since DEspR overlaps with a larger transcript, FBXW7, on the opposite strand [3], RNA-seq databases that specifically exclude double stranded DNA [13] will also exclude RNA-RNA sequences such as DEspR-FBXW7 hybrids. The process-exclusion of RNA-RNA hybrids in RNA-Seq entries in current methodologies is due to the standard of excellence requiring elimination of all double stranded DNA in RNA-Seq libraries [13], which inadvertently will also exclude RNARNA hybrids. More specifically, since RNA-RNA hybrids can form in standard RNA isolation methods with phenol extraction [14], and can form during RNA-seq library preparation using primer annealing reactions at temperatures lower than the melting temperatures (Tm) of RNARNA hybrids, and given process-exclusion of any dsDNA or RNA-RNA or dsDNA-RNA hybrids, there is an inadvertent bias introduced against the detection of DEspRspecific transcripts due to RNA-RNA hybrids formed between DEspR transcript and exon\#5 (or \#6 depending on dataset numbering) of FBXW7 transcript on the opposite strand.

In parallel, the non-detection in proteomic databases is concordant with difficulties in detecting membrane proteins with single transmembrane domains that are less than $150 \mathrm{kDa}$ [15], especially if glycosylated, since PNGase F treatments do not necessarily ensure detection by MS [16]. The current non-detection of DEspR transcripts in transcriptomics could also be due to the exclusion of RNA-RNA hybrids, and in primer pair-specific amplification due to the absence of DEspR-specific primers given the non-recognition of the DEspR gene by NCBI.

\section{Confirmation of translatability}

Demonstration of translatability through different experimental designs and using anti-human-DEspR mAbs 
a

Live cell imaging of Panc1 tumor cells
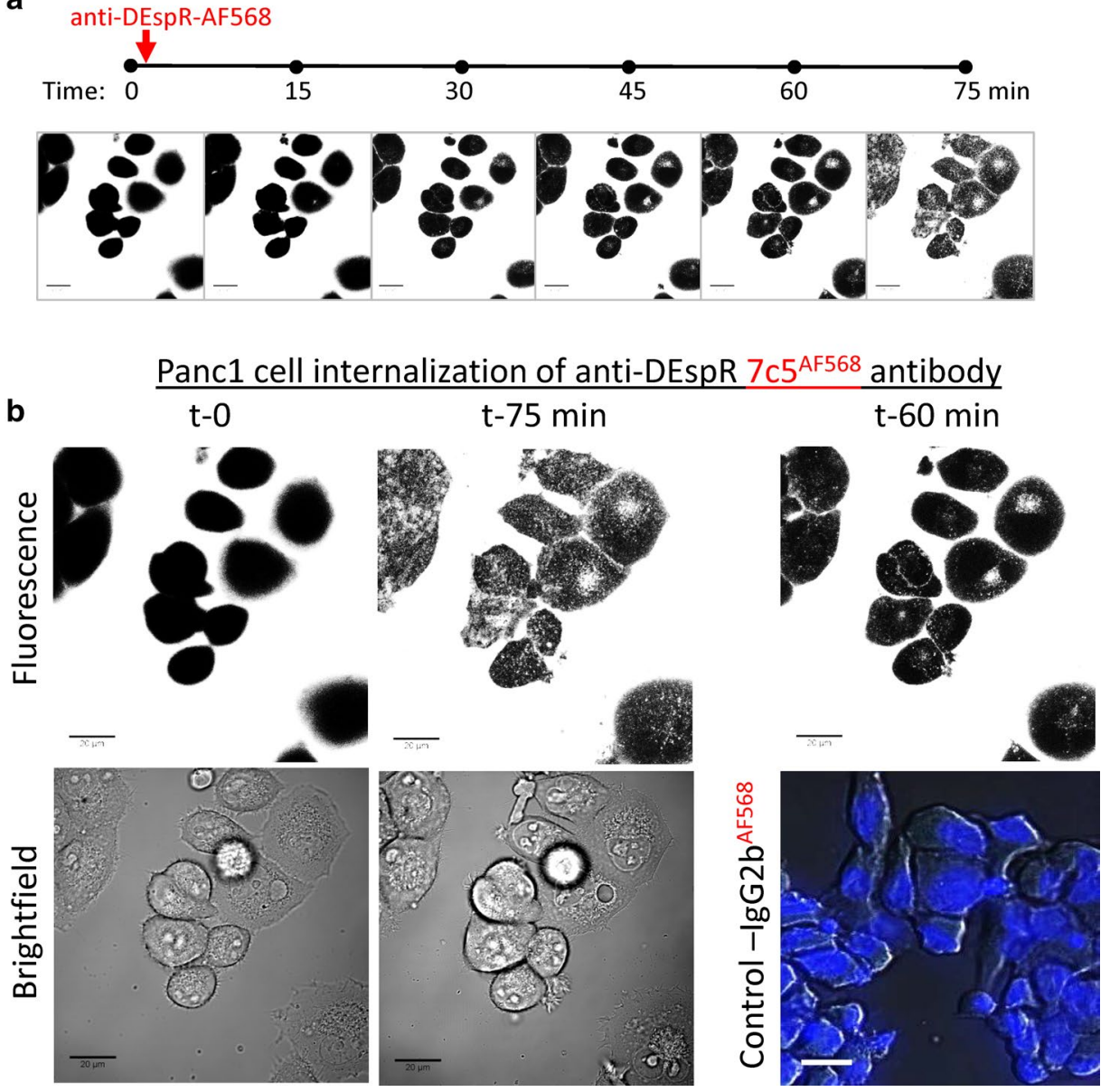

Fig. 5 Representative time series of internalization of fluorescently labeled (AF568) anti-DEspR 7c5b2-mAb by Panc1 tumor cells within $1.5 \mathrm{~h}$. a Confocal images showing representative Panc1 tumor cells from baseline (t-0) prior to addition of AF568-labeled antibody, up to $1 \mathrm{~h}, 15 \mathrm{~min}$ from addition of AF568-7c5b2 mAb. Increasing intracellular fluorescence (white) is detected in multiple Panc1 cells. b Higher magnification of Panc1 tumor cells at baseline and t-75 min with corresponding bright field images of Panc1 cells. At t-60 min, representative image of Panc1 tumor cells exposed to control AF568-labeled lgG2b isotype, with DAPI stained nuclei (blue) to mark cells, demonstrate no intracellular AF568 fluorescence (red) uptake. Bar $=20$ microns

specific for different epitopes substantially fulfill UNIPROT criteria for determining a protein's existence at the highest level such as detection of protein by antibody, demonstration of protein-protein interactions, demonstration of post-translational modifications, and demonstration of protein functionality [8].

Pull-down protein analysis experiments confirm the existence of DEspR via direct detection of the protein in human tumor cells by Western blot analysis of DEspR using multi-epitope anti-DEspR mAbs. The detection of the identical protein band by Western blot 'walking' with distinct anti-DEspR mAbs targeting two different epitopes, $5 \mathrm{~g} 12 \mathrm{e} 8 \mathrm{mAb}$ binding to DEspR epitope- 1 that spans a splice junction, and 6g8g7 binding to DEspR epitope 2 that spans tryptophan[TGG]-codon\#14, demonstrate the existence of the DEspR protein. If the stop codon were indeed present at codon\#14 instead of tryptophan, there would simply be a 13 amino acid peptide with $1.5 \mathrm{kDa} \mathrm{MW}$ rather than the glycosylated 17.5 and $12.5 \mathrm{kDa}$ protein bands, or the non-glycosylated DEspRsequence predicted $\sim 10 \mathrm{kDa}$ protein.

Moreover, if there were indeed a stop codon at codon\#14, DEspR would not be glycosylated nor internalized as the consensus sequence motifs for these sites are beyond the reported stop codon\#14. The detection of the identical-sized protein on Western blot analysis of DEspR-positive Cos1 cell permanent transfectants and U87 tumor cells corroborates the protein product of the 
transfected plasmid DEspR mini-gene construct previously reported, and corroborates splicing of the unspliced DEspR cDNA mini-gene construct [2].

\section{Detection of DEspR by Western blotting but not by mass spectrometry analysis}

We note that although DEspR was detected consistently in denatured conditions via Western blot analysis of membrane protein pull-down products using $5 \mathrm{~g} 12 \mathrm{e} 8$ and 6g8g7 anti-DEspR mAbs, MS did not detect DEspR-specific peptides. However, non-detection by MS does not negate DEspR protein existence for several reasons. First, based on deduced amino acid sequence predicting a single transmembrane integral membrane $\sim 10 \mathrm{kDa}$ protein that is glycosylated to $17.5 / 12.5 \mathrm{kDa}$, non-detection by peptide mass fingerprinting methods using techniques such as MALDI-TOF MS analysis is not surprising given that only 204 integral membrane proteins were detected on mass spectrometry in a study of rat endothelial cells at the National Center for Proteomics Research, and that no VEGF receptor-2 $(151 \mathrm{kDa})$ was detected in this study [17]. Using 6718 as total number of membrane proteins [18], Mirza et al. [17] detected $3 \%$ integral membrane proteins on MS, while Peng et al. [19] detected 301 integral membrane proteins via SDS-PAGE shotgun proteomics or $4.5 \%$ of 6718 total membrane proteins, and highest count using SCX-RPLC-MS/MS (MudPIT) strategy detected 876 integral membrane proteins or $13 \%$ of 6718 integral membrane proteins in murine NK cells [20]. Notably, of the ones detected by MS using an improved method that detects more membrane proteins using a centrifugal proteomic reactor, all the proteins detected were $>150 \mathrm{kDa}$ and $\geq 2$ transmembrane domains [15].

Other factors could also account for non-detection by MS with current methodologies. As stated by Bensalem et al. [21], peptide mass fingerprinting of membrane proteins, using techniques such as MALDI-TOF MS, remains a significant challenge for at least three reasons: (1) membrane proteins are naturally present at low levels, (2) many detergents strongly inhibit proteases and have deleterious effects on MALDI spectra, and (3) despite the presence of detergent, membrane proteins are unstable and often aggregate [21]. Additionally, glycosylation of DEspR also impedes current proteomic detection as reported by Cao et al. [16] stating that "deglycosylation of plasma membrane proteins by treatment with PNGaseF did not yield detection of additional hydrophobic proteins". Therefore, peptide mass fingerprinting MS analysis of PNGase-treated pull-down proteins which did not identify DEspR does not negate the existence of DEspR protein. These observations by others account for the non-detection by MS of the single transmembrane receptor $<150 \mathrm{kDa}$ DEspR protein, thus excluding MS non-detection as counter evidence for the existence of DEspR protein, especially given multiple other experimental evidence that fulfill UNIPROT criteria for determining a protein [8].

\section{Demonstration of protein interactions, protein-specific properties and functionality}

Aside from ligand-specific activated DEspR signaling [3], detection of DEspR-protein interactions in the DEspRGalectin-1 complex, along with other pull-down protein partners, complies with a UNIPROT criterion for ascertaining existence of a protein as a gene product through the demonstration of protein-interactions [8]. The distinct functionality of DEspR vs Galectin-1 in terms of anoikis resistance indicates DEspR protein-specific functions, rather than the potential counter-argument that DEspR functionality is merely due to the known Galectin-1 functions.

More specifically, DEspR-specific inhibition by blocking antibodies induces anoikis in four cancer tissue type CSCs, corroborating earlier observations for Panc1 and U87 CSCs [3]. In contrast, Galectin-1 is pro-anoikis [22]. Given these two observations, we hypothesize that glycosylated DEspR plays a role in anoikis resistance by binding to Galectin-1 and inhibiting its pro-anoikis function, much like glycosylation of p16-INK4a de-induces Galectin-1 pro-anoikis functions through upregulation of Galectin-3 [22]. Confirmation of the DEspR-Galectin-1 complex is demonstrated by co-localization on doubleimmunostaining experiments, thus further strengthening evidence for translatability and functionality by the detection of protein-protein interactions following UNIPROT criteria [8]. Distinguishing the DEspR protein band by size and quantity from its pull-down partners identified from MS by their corresponding antibodies in serial sequential probing of the identical western blot, corroborates their detection by MS, as well as eliminates the potential of erroneous cross-reactivity pull-down results.

More importantly, given that demonstration of protein functionality certifies a protein's existence $[7,8]$, the demonstration of DEspR expression in $>50 \%$ of CSCs in four different cancer tissue types by FACS analysis and DEspR functionality via blocking antibody inhibition of CSC growth in all four different cancer tissue type CSCsglioblastoma, triple negative breast cancer, non-small cell lung cancer and pancreatic ductal adenocarcinoma, provide compelling evidence of the existence of DEspR protein based on UNI-PROT criteria and standards in the field $[7,8]$.

Furthermore, the demonstration of internalization of DEspR protein via live cell imaging is consistent with a 
consensus internalization recognition sequence downstream to amino acid\#14. This not only confirms translatability of the DEspR protein beyond 13 amino acids, thus refuting the stop codon presence, but also demonstrates a protein-specific property, i.e. receptor internalization and expands DEspR's multi-functionality. Concordantly, these new data on functionality are supported by previous findings of DEspR roles in tumor angiogenesis, invasiveness, growth, as well as CSC survival in adverse conditions [3].

\section{Conclusions}

Altogether, in the context of an error prone site at codon\#14, multiple protein assays independently and collectively demonstrate the existence of the DEspR protein, and analyses that show DEspR protein properties (protein-protein interactions, glycosylation, internalization) and functional roles in multiple cancer tissue type CSCs, collectively certify the DEspR protein based on multiple UNIPROT criteria and demonstrate that the human DEspR gene is not a pseudogene. Given that fulfillment of a single UNIPROT criterion for a protein's existence is sufficient, fulfillment of multiple criteria present compelling experimental evidence, at the highest protein level, that functional human DEspR protein exists.

\section{Methods}

\section{Maxam-Gilbert sequencing}

We performed Maxam-Gilbert sequencing essentially as described [23]. The hDEspR cDNA was $5^{\prime}$-end labelled with polynucleotide kinase using gamma- ${ }^{32} \mathrm{P}$ ATP. Chemical treatments for Guanine (G), Guanine + Adenine $(\mathrm{G}+\mathrm{A})$, Cytosine + Thymine $(\mathrm{C}+\mathrm{T})$ and Cytosine $(\mathrm{C})$ cleavages were done as described [23]. Cleavage products from the four reactions were size separated by $8 \%$ denaturing polyacrylamide gel electrophoresis and fragments visualized by autoradiography. Electrophoresis was performed at three different fixed wattages: 25 watts (\#1), 35 watts (\#2) and 50 watts (\#3).

\section{Cell lines and antibody development and characterization by ELISA}

Verified glioblastoma U87 MG (cat\# ATCC HTB-14), triple negative breast cancer MDA-MB-231 (cat\# ATCC HTB-26), non-small cell lung cancer NCI-H460 (cat\# ATCC HTB-177) and pancreatic cancer Panc-1 (cat\# ATCC CRL-1469) cell lines were obtained from ATCC. Isolation and propagation of U87, MB-231, H460 and Panc1 cancer stem cell-like cells (CSCs) was done as described [3]. CSCs were maintained and expanded through passage-5 in complete MammoCult ${ }^{\circledR}$ medium (Stem Cell Technologies, BC, Canada) containing $0.5 \%$ Methylcellulose (Stem Cell Technologies, BC, Canada) in
$100 \mathrm{~mm}$ ultra-low attachment plates in $5 \% \mathrm{CO} 2$ humidified incubator at $37{ }^{\circ} \mathrm{C}$. Testing for increased tumorigenicity was performed in vivo at passage-5. Monoclonal antibody development was custom performed by ProMab Biotechnologies (Richmond, CA). For 7c5b2 [3] and $5 \mathrm{~g} 12 \mathrm{e} 8 \mathrm{mAbs}$ we used a nine amino-acid peptide, $\mathrm{M}_{1}$ TMFKGSN $\mathrm{E}_{9}$ at the amino-terminal end of hDEspR [2] and a ten amino-acid peptide, $\mathrm{E}_{9} \mathrm{MKSRWNWGS}_{18}$, for $6 \mathrm{~g} 8 \mathrm{~g} 7 \mathrm{mAb}$ as antigens respectively. Screening of hybridoma supernatants and characterization of monoclonal antibodies were performed by ELISA using corresponding antigenic peptides. Serial dilutions of primary antibodies were incubated at $37{ }^{\circ} \mathrm{C}$ for $1 \mathrm{~h}$. The wells were then incubated with horse radish peroxidase (HRP)labeled anti-IgG (Sigma) at $37{ }^{\circ} \mathrm{C}$ for $1 \mathrm{~h}$. Reactions were analyzed at $450 \mathrm{~nm}$ after addition of 3,3'5,5'-tetramethylbenzidine substrate at $37^{\circ} \mathrm{C}$ for $10 \mathrm{~min}$.

\section{Magnetic bead immunoprecipitation (pull-down) of DEspR protein-complex}

U87 CSC membranes, Cos1-hDEspR permanent cell transfectant membranes and U87 xenograft tumor (in nude rats) membranes were isolated by differential centrifugation as described [2]. Antibody coupling to magnetic Dynabeads M-450 Epoxy (Invitrogen) was performed as per manufacturer's instructions using $200 \mu \mathrm{g}$ of anti-hDEspR $5 \mathrm{~g} 12 \mathrm{e} 8 \mathrm{mAb}$ per $1 \mathrm{~mL}$ of beads $\left(4 \times 10^{8}\right.$ beads). For target binding $100 \mu \mathrm{L}$ of $5 \mathrm{~g} 12 \mathrm{e} 8$-coupled beads $\left(4 \times 10^{7}\right.$ beads $)$ and $1.3 \mathrm{mg}$ membrane protein were incubated for $2 \mathrm{~h}$ at $4{ }^{\circ} \mathrm{C}$ in $1 \mathrm{ml}$ ice-cold PBS containing $2 \mathrm{mM}$ EDTA. To capture mAb-bound protein, the tube was placed in a magnet for $2 \mathrm{~min}$, the supernatant discarded and the beads washed five times with $1 \mathrm{~mL}$ PBS buffer containing $2 \mathrm{mM}$ EDTA and $0.2 \%$ Tween- 20 at room temperature. This washed magnetic bead-'pulldown'-protein complex was then analyzed using different methods. (a) For SDS-PAGE (18\% polyacrylamide) the beads were resuspended in $20 \mu \mathrm{L}$ of $1 \mathrm{X}$ Laemmle sample buffer and proteins denatured at $65^{\circ} \mathrm{C} \times 30 \mathrm{~min}$ in order to size-separate and assess size(s) of protein(s) 'pulleddown. (b) For PNGase F (SIGMA) treatment of pulldown proteins to assess glycosylation states of proteins pulled-down, the beads were resuspended in $20 \mu \mathrm{L}$ of a buffer containing $20 \mathrm{mM}$ ammonium bicarbonate $\mathrm{pH} 8.0$, $1 \%$ SDS, $100 \mathrm{mM}$ beta-mercaptoethanol and incubated for $30 \mathrm{~min}$ at $65^{\circ} \mathrm{C}$. The tube was then placed in a magnet for $2 \mathrm{~min}$. The supernatant was collected to which $20 \mu \mathrm{L}$ of $0.25 \mathrm{M} \mathrm{KCl}$ was added to eliminate SDS by precipitation. To lower $\mathrm{KCl}$ concentration the supernatant $(40 \mu \mathrm{L})$ was collected, diluted $10 \mathrm{X}$ with $20 \mathrm{mM}$ ammonium bicarbonate $\mathrm{pH} 8.0$ and concentrated by using a Microcon YM-3 centrifugal filter (Millipore). The concentrated sample was treated with PNGase F (2.5 units) in a final 
volume of $20 \mu \mathrm{L}$ for $3 \mathrm{~h}$ at $37^{\circ} \mathrm{C}$. Gels were stained either with QC Colloidal Coomassie Stain (Bio-Rad) or Silver (BioRad Silver Stain Plus Kit) following manufacturer's instructions.

\section{Western blot analysis}

Western blot analysis was done essentially as described [2] using pull-down proteins extracted from $2 \times 10^{7}$ 5g12e8-coupled Dynabeads. Serial Western blot analyses of proteins pulled down-hDEspR, hGalectin-1, hRab$1 \mathrm{~b}$ and hTMED10-were done sequentially by stripping and re-probing the same blot in the following order: 1st, human-specific DEspR mAb (5g12e8 $40 \mu \mathrm{g} / \mathrm{ml}$, secondary anti-mouse IgG at 1:20,000), 2nd, Rab-1b polyclonal antibody (pAb) (Santa Cruz Biotechnology, cat \# sc-599, at $1 \mu \mathrm{g} / \mathrm{ml}$, secondary anti-rabbit IgG at 1:20,000), 3rd, human-specific Galectin-1 (Abcam, cat \# ab25138 at $0.4 \mu \mathrm{g} / \mathrm{ml}$, secondary anti-rabbit IgG $1: 20,000$ ) and 4 th, pAb TMED10 (Abcam, cat \# ab72666, at $10 \mu \mathrm{g} / \mathrm{ml}$, secondary anti-rabbit IgG 1:20,000).

Serial Western blot analyses of U87 tumor cell membrane and Cos1-hDEspR cell membrane pull-downs were done sequentially by stripping and re-probing the same blot in order: 1st, hDEspR mAb $(5 \mathrm{~g} 12 \mathrm{e} 840 \mu \mathrm{g} / \mathrm{ml}$, secondary anti-mouse IgG at 1:20,000) and then 2nd, hGalectin-1 (Abcam, cat\#ab25138, at $0.4 \mu \mathrm{g} / \mathrm{ml}$, secondary anti-rabbit IgG 1:20,000).

Serial western blot of U87 tumor membrane pull-down was also reacted sequentially by stripping and re-probing the same blot using first the following in order: 1st, $\mathrm{hDE}$ spR mAb $(6 g 8 g 740 \mu \mathrm{g} / \mathrm{ml}$, secondary anti-mouse IgG at 1:20,000), and 2nd, hDEspR mAb (5g12e $840 \mathrm{ug} / \mathrm{ml}$, secondary anti-mouse IgG at 1:20,000).

Immunoreactive proteins were detected by chemiluminescence using the ECL Western Detection kit (GE Healthcare) as per manufacturer's specifications.

\section{Mass spectrometry analysis}

Mass spectrometry of pull-down proteins was customperformed by Creative Proteomics (Shirley, NY). Briefly, proteins were reduced for $40 \mathrm{~min}$ with $5 \mathrm{mM}$ dithiothreitol in $25 \mathrm{mM} \mathrm{NH} 4 \mathrm{HCO} 3$ at room temperature and alkylated for $40 \mathrm{~min}$ with $15 \mathrm{mM}$ iodoacetamide in $25 \mathrm{mM}$ NH4HCO3 in the dark. After washed and dehydrated, the alkylated samples were digested overnight at $37{ }^{\circ} \mathrm{C}$ with trypsin in a $1: 50$ enzyme-to-substrate ratio (Promega, V5113). Following digestion, the peptide mixtures were acidified with trifluoroacetic (TFA) to $1 \%$, and desalted by home-made C18 tips. Finally, the desalted peptide samples were dried and dissolved in $10 \mu \mathrm{L}$ of $0.1 \%$ formic acid in water and subjected to nanoLC-MS/ MS analysis in a Q Exactive mass spectrometer. The raw MS files were analyzed and searched against Uniprot human protein sequence database using Proteome Discoverer 1.4 (Thermo Fisher Scientific, USA). The parameters were set as follows: the protein modifications were carbamidomethylation (C) (fixed), oxidation (M) (variable), and myristyl on glycine (variable), Asn to Asp (variable); the enzyme specificity was set to trypsin; the maximum missed cleavages were set to 2; the precursor ion mass tolerance was set to $10 \mathrm{ppm}$, and MS/MS tolerance was 0.6 Da.

\section{Immunofluorescence analysis of subQ xenograft U87-CSC tumors}

Double immunofluorescence staining was done as described [3]. Human-specific anti-DEspR mAb (5g12e8) and human-specific anti-Galectin-1 antibody (Abcam, cat\#ab25138) were labeled with AlexaFluor(AF)-488 or AF568, and used at $1 \mu \mathrm{g} / \mathrm{ml}$ for anti-Galectin- 1 and at $100 \mu \mathrm{g} / \mathrm{ml}$ for $5 \mathrm{~g} 12 \mathrm{e} 8 \mathrm{mAbs}$ on fixed, paraffin-embedded sections following antigen-retrieval. Digital photomicroscopy was done using a Zeiss Axioskop fluorescence microscope with auto-exposure settings.

\section{FACS analysis of U87-CSCs, MB-231-CSCs, H460-CSCs and Panc1-CSCs}

U87-CSCs, MB-231-CSCs, H460-CSCs and Panc1-CSCs were incubated in ice-cold Hank's balanced salt solution (HBSS, Invitrogen, NY) plus $2 \%$ FBS containing: (a) $10 \mu \mathrm{g} /$ $\mathrm{ml}$ AF-568 labeled 7c5b2 mAb, or (b) $10 \mu \mathrm{g} / \mathrm{ml} \mathrm{AF-568}$ labeled IgG2b as isotype control. Duplicate samples were incubated for $20 \mathrm{~min}$ at $4{ }^{\circ} \mathrm{C}$, washed, resuspended in $1 \%$ FBS/HBSS, 1 \% PFA, filtered and analyzed on an LSR-IIFACS instrument. Analysis was done using FloJo Flow Cytometry Analysis Software (http://www.FloJo.com).

\section{DEspR-inhibition of CSC-growth}

DEspR-inhibition studies were performed as described [3]. CSCs (2000/well) were seeded in ultra-low attachment 96-well plate and treated with different blocking anti-hDEspR mAbs (5g12e8, 7c5b2 and 6g8g7) at $100 \mu \mathrm{g} /$ $\mathrm{ml}$, compared with control non-treated CSCs with six replicates for each. CSCs were cultured in optimal (5\% $\mathrm{CO} 2$, humidified incubator at $37^{\circ} \mathrm{C}$ ) non-adherent conditions. Anti-hDEspR (5g12e8, 7c5b2 and 6g8g7) mAbs were added at seeding, day- 2 and day-4. Live and dead CSCs were counted using Trypan Blue on day- 5 .

\section{RNA sequence analysis}

The NCBI Sequence Read Archive was searched on $7 / 17 / 2013$ with a query sequence provided by the Genbank accession for "Homo sapiens dual endothelin1(VEGFsp)/angiotensin II receptor (DEAR) mRNA, complete cds," gi|144,954,325|gb|EF212178.1|, against 727 sequencing runs (Additional file 5). 


\section{Live cell imaging}

Fluorescence and transmitted light images were acquired with a Zeiss LSM 710 Duo confocal microscope (Carl Zeiss, Thornwood NY). Excitation was from a $561 \mathrm{~nm}$ DPSS laser. A 63X 1.4 NA planapochromat oil immersion objective was used. The cells were in $35 \mathrm{~mm}$ dishes with coverglass bottoms. Emission was collected from 575-725 nm. Cells were maintained under physiological conditions using a Pecon stage-top incubation system which maintained $37^{\circ}$ centigrade and $5 \% \mathrm{CO} 2$. Analysis was done using Image J (Image J, Wayne Rasband, NIH) with identical settings for brightness and contrast adjustments for all images. Live cell imaging with AF-568 labeled isotype IgG2b control was performed in identical conditions. At $60 \mathrm{~min}$, cells were fixed briefly and mounted with Vectashield mounting medium with DAPI (Vector Laboratories, CA, Cat.\# H-1200). Photomicroscopy was done using a Zeiss Axioskop fluorescence microscope (Carl Zeiss, Thornwood $\mathrm{NY}$ ) with differential interference contrast (DIC).

\section{Statistical analysis}

All data were analyzed for normality and descriptive statistics. The following statistical tests were performed using SigmaPlot 11.0 or PRISM 5: one-way analysis of variance (ANOVA) followed by Tukey multiple comparisons test (MCT) for CSC-growth inhibition experiments. A $P<0.05$ was considered statistically significant.

\section{Additional files}

Additional file 1: Figure S1. NCBI designation of Dear (alias DEspR) gene as pseudogene-updated May 2016.

Additional file 2: Figure S2. Sanger dideoxy-sequencing of DEspR spanning amino acid 14 (aa14) codon-TGG position within a Yamakawa compression-motif [Y-G-N1, 2-A-R]: DEspR 5' T-G-G-A-A, shows two G's (GG), and downward slippage/compression of the two G's towards the 5' T. A 3-nt long stem-loop structure spans the compression motif region in human DEspR but not in rat DEspR.

Additional file 3: Figure S3. Unedited RNA-Seq entries showing the questioned two G's with insertion A or TA document difficult sequencing region as seen in manual sequencing.

Additional file 4: Table S1. Protein profile pulled-down (Creative Proteomics). Proteins pulled-down with anti-DEspR mAb 5g12e8 from U87 CSC membranes.

Additional file 5. RNA-seq analysis. List of 727 sequencing runs searched with a DEspR query sequence in the NCBI Sequence Read Archive.

\footnotetext{
Abbreviations

DEspR: dual endotheiln-1/NEGFsp receptor; VEGFsp: vascular endothelial growth factor signal peptide; CSC: cancer stem-like cell; ARMS: allele-specific amplification-refractory mutation system; ORF: open reading frame; FBXW7: F-box and WD repeat domain containing 7; mAb: monoclonal antibody; Gal-1: galectin-1; Gal-3: galectin-3; TMED10: transmembrane emp24 domain-containing protein 10; Rab-1b: ras-related protein Rab-1B; PNGase F: peptide-Nglycosidase F; MS: mass spectrometry.
}

\section{Authors' contributions}

VLMH wrote the manuscript, co-supervised experimental design and data analysis, performed immunofluorescence analysis, co-supervised FACS analyses; MS performed RNA-seq analysis and revised manuscript; AMM performed Maxam-Gilbert sequencing, pull-down experiments and western blot analysis; GT performed pull-down experiments, western blot analysis, FACS analysis and DEspR inhibition of CSC-growth; KAP performed FACS analysis; KR and DJP performed mass spectrometry analysis; DJP advised on pull-down experiments and analysis; NR-O designed the experiments, supervised the studies, performed all statistical analyses and contributed to writing the manuscript. All authors read and approved the final manuscript.

\section{Author details}

${ }^{1}$ Whitaker Cardiovascular Institute, Boston University School of Medicine, 700 Albany Street, Boston, MA 02118, USA. ${ }^{2}$ Department of Medicine, Boston University School of Medicine, 700 Albany Street, Boston, MA 02118, USA. ${ }^{3}$ Department of Pathology and Biomedical Engineering, Boston University, Boston, USA. ${ }^{4}$ Cold Spring Harbor Laboratory, 1 Bungtown Road, Cold Spring Harbor, NY 11724, USA.

\section{Acknowledgements}

We thank Francis J. Carr, Ph.D. for helpful discussions and manuscript editing; and Michael T. Kirber, Ph.D. for Live Cell Imaging in the Evans Department of Medicine Cell Imaging Core.

\section{Availability of data and material}

The datasets supporting the conclusions of this article are included within the article and its additional files.

\section{Competing interests}

Boston University has filed patents on DEspR for diagnostic and therapeutic agents with $\mathrm{NRO}$ and VLMH as co-inventors.

\section{Funding}

This work was partially supported by Ignition Awards from BU Office of Technology Development, and CTSI Award from BU-Clinical and Translational Science Institute Grant NIH-U54TR001012. Some Mass Spectrometry analyses were performed at Cold Spring Harbor Laboratory Shared Resources, which are funded by the Cancer Center Support Grant 5P30CA045508. The funding body had no role in design, in the collection, analysis, and interpretation of data; in the writing of the manuscript; and in the decision to submit the manuscript for publication.

Received: 28 January 2016 Accepted: 20 May 2016

Published online: 14 June 2016

\section{References}

1. NCBI.gov. DEAR dual endothelin-1(VEGFsp)/angiotensin II receptor pseudogene [Homo sapiens (human)]. http://www.ncbi.nlm.nih.gov/ gene/102191832. Accessed 27 Aug 2015.

2. Glorioso N, Herrera VL, Bagamasbad P, Filigheddu F, Troffa C, Argiolas $\mathrm{G}$, et al. Association of ATP1A1 and Dear SNP-haplotypes with essential hypertension: sex-specific and haplotype-specific effects. Circ Res. 2007;100:1522-9.

3. Herrera VL, Decano JL, Tan GA, Moran AM, Pasion KA, Matsubara Y, et al. DEspR roles in tumor vasculo-angiogenesis, invasiveness, CSC-survival and anoikis resistance: a 'common receptor coordinator' paradigm. PLoS ONE. 2014;9(1):e85821.

4. Meacham F, Boffelli D, Dhahbi J, Martin DIK, Singer M, Pachter L. Identification and correction of systematic error in high-throughput sequence data. BMC Bioinform. 2011;12:451.

5. Brodin J, Mild M, Hedskog C, Sherwood E, Leitner T, Andersson B, et al. PCR-induced transitions are the major source of error in cleaned up ultradeep pyrosequencing data. PLoS One. 2013;8(7):e70388.

6. Pei B, Sisu C, Frankish A, Howald C, Habegger L, Mu XJ, et al. The GENCODE pseudogene resource. Genome Biol. 2012;13:R51. 
7. Kageyama Y, Kondo T, Hashimoto Y. Coding vs non-coding: translatability of short ORFs found in putative non-coding transcripts. Biochimie. 2011;93:1981-6.

8. Uni-Prot.org. http://www.uniprot.org. Accessed 24 Oct 2015.

9. Yamakawa $H$, Nakajim D, Ohara O. Identification of sequence motifs causing band compressions o human cDNA sequencing. DNA Res. 1996;3:81-6.

10. Camby I, Mercier ML, Lefranc F, Kiss R. Galectin-1: a small protein with major functions. Glycobiology. 2006;16:137R-57R.

11. Wong JJ, Ritchie W, Ebner OA, Selbach M, Wong JWH, Huang Y, et al. Orchestrated intron retention regulates normal granulocyte differentiation. Cell. 2013;154:583-95.

12. Wong JJ, Au AY, Ritchie W, Rasko JE. Intron retention in mRNA: no longer nonsense: known and putative roles of intron retention in normal and disease biology. Bioessays. 2016;38:41-9.

13. Levin JZ, Yassour M, Adiconis X, Nusbaum C, Thompson DA, Friedman $\mathrm{N}$, et al. Comprehensive comparative analysis of strand-specific RNA sequencing methods. Nat Methods. 2010;7:709-15.

14. Faridani OR, Mclnerney GM, Gradin K, Good L. Specific ligation to doublestranded RNA for analysis of cellular RNA:RNA interactions. Nucleic Acids Res. 2008;36:e99.

15. Zhou H, Wang F, Wang Y, Ning Z, Hou W, Wright TG, et al. Improved recovery and identification of membrane proteins from rat hepatic cells using a centrifugal proteomic reactor. Mol Cell Proteomics. 2011;10:1-11.

16. Cao L, Clifton JG, Reutter W, Josic D. Mass spectrometry-based analysis of rat liver and hepatocellular carcinoma Morris hepatoma 7777 plasma membrane proteome. Anal Chem. 2013;85:8112-20.
17. Mirza SP, Halligan BD, Greene AS, Olivier M. Improved method for the analysis of membrane proteins by mass spectrometry. Physiol Genomics. 2007;30:89-94

18. Almén MS, Nordström KJ, Fredriksson R, Schiöth HB. Mapping the human membrane proteome: a majority of the human membrane proteins can be classified according to function and evolutionary origin. BMC Biol. 2009;7:50-63.

19. Peng L, Kapp EA, McLauhlan D, Jordan TW. Characterization of the Asia Oceania human proteome organization membrane proteomics initiative standard using SDS-PAGE shotgun proteomics. Proteomics. 2011:11:4376-84.

20. Fagerberd L, Jonasson $K$, vonHeijne G, Uhlen M, Berglund L. Prediction of the human membrane proteome. Proteomics. 2010;10:1141-9.

21. Bensalem N, Masscheleyn S, Mozo J, Vallee B, Brouillard F, Trudel S, et al. High sensitivity identification of membrane proteins by MALDI TOF-MASS Spectrometry using polystyrene beads. J Proteome Res. 2007;6:1595-602.

22. Sanchez-Ruderisch H, Fischer C, Detjen KM, Welzel M, Wimmel A, Maing JC, et al. Tumor suppressor p16 INK4a: downregulation of galectin-3, an endogenous competitor of the pro-anoikis effector galectin-1, in a pancreatic carcinoma model. FEBS J. 2010;277:3552-63.

23. Maxam AM, Gilbert W. A new method for sequencing DNA. Proc Natl Acad Sci USA. 1977:74:560-4.

24. Ruiz-Opazo N, Hirayama K, Akimoto K, Herrera VL. Molecular characterization of a dual endothelin-1/angiotensin II receptor. Mol Med. 1998:4:96-108

\section{Submit your next manuscript to BioMed Central and we will help you at every step:}

- We accept pre-submission inquiries

- Our selector tool helps you to find the most relevant journal

- We provide round the clock customer support

- Convenient online submission

- Thorough peer review

- Inclusion in PubMed and all major indexing services

- Maximum visibility for your research

Submit your manuscript at www.biomedcentral com/submit
O Biomed Central 